\title{
BIM METHODOLOGY APPLIED TO THE PREPARATION OF MANAGEMENT CONSTRUCTION
}

\section{Marcus Cruz dos Santos ${ }^{1}$, Renan Diego Amanajás Lima ${ }^{2}$}

${ }^{1,2}$ Discente de Engenharia Civil do Centro Universitário do Norte (UNINORTE), Av. Igarapé de Manaus, 211, Centro, ManausAM

Email: santos.m.0752@gmail.com, renan.amanajas@gmail.com.

Received: April 11'th, 2019

Accepted: May 21 ${ }^{\text {th }}, 2019$

Published: June 30th, 2019

Copyright (C)2016 by authors and Institute of Technology Galileo of Amazon (ITEGAM). This work is licensed under the Creative Commons Attribution International License (CC BY 4.0). https://creativecommons.org/lice nses/by/4.0/

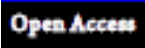

\section{ABSTRACT}

The BIM platform is a work philosophy that integrates architects, engineers and builders in the elaboration of an accurate virtual model, which generates a database containing both topological information and the necessary subsidies for budgeting, energy calculation and forecasting. phases of construction. BIM works as a database manager, that any changes or revisions made to any part of a project will be automatically modified in all other forms of visualization, whether they are tables, reports or drawings (documents), generated from the model. With the use of the BIM platform and using the tools included in it, for example, REVIT, NAVISWORK, ARCHICAD, CYPE 3D, MSPROJECT, design, construction and operation can be done much faster, more efficiently and economically. In this article, a comparison was made between two companies, where company A will not use the BIM platform and it is entire project, only in the budget. Company B, BIM systems are used for the modeling of projects and generation of views, cuts, facades, all documentation in a more detailed and with better quality and better visualization, but it does not take advantage of the various advantages offered by the system. After the comparison made between companies $\mathrm{A}$ and $\mathrm{B}$, it will be shown that the lack of professionals qualified to use the BIM platform is the reason for the delay in the use of this technology and that the use in both companies is still low, due to the focus of company $\mathrm{B}$, which did not reach the construction site, and the lack of professionals trained in company A, who had difficulty in using it.

Keywords: BIM, Revit, Project.

\section{METODOLOGIA BIM APLICADA À PREPARAÇÃO E GESTÃ̃ DE OBRA RESUMO}

A plataforma BIM é uma filosofia de trabalho que integra arquitetos, engenheiros e construtores, na elaboração e execução de um modelo virtual preciso do projeto, o qual gera uma base de dados que contém tanto informações topológicas como os subsídios necessários para orçamento, cálculo energético e previsão das fases da construção. O BIM trabalha como gestor de banco de dados, de forma que qualquer alteração ou revisão realizada em qualquer parte de um projeto será automaticamente modificada em todas as demais formas de visualização, sejam tabelas, relatórios ou desenhos (documentos), gerados a partir do modelo. Com o uso da plataforma BIM e usando as ferramentas nela inclusa como por exemplo, REVIT, NAVISWORK, ARCHICAD, CYPE 3D, MSPROJECT, o projeto, a construção e a operação podem ser feita de modo muito mais rápido, eficiente e econômico. No presente artigo, foi feito a comparação entre 2 empresas, onde a empresa A não utilizará a plataforma BIM e todo seu projeto, apenas no orçamento. A empresa B, os sistemas BIM são utilizados para a modelagem dos projetos e geração de vistas, cortes, fachadas, toda documentação de forma mais detalhada e com maior qualidade e melhor visualização, porém não são aproveitas as diversas vantagens oferecidas pelo sistema. Após a comparação feita entre as empresas A e B, será mostrado que a falta de profissional habilitado para a utilização da plataforma BIM é a causa para o atraso do avanço do uso dessa tecnologia e que a utilização em ambas empresas ainda é baixa, pelo foco da empresa $\mathrm{B}$, que não chegava ao canteiro de obras, e pela falta de profissionais treinados na empresa A, que obteve maior dificuldade no uso.

Keywords: BIM, Revit, Projeto. 


\section{INTRODUÇÃO}

O avanço tecnológico vem se desenvolvendo cada vez mais no setor da Engenharia Civil, revolucionando todo o segmento da indústria da construção civil. O uso de softwares CAD (Computer Aided Design - Desenho Assistido por Computador), que se tornou popular na década de 80 , vieram para mudar os paradigmas de projeto e auxiliarem na evolução tecnológica da construção civil.

Existem muitas verdades e mitos na percepção geralmente aceita, no que se refere ao estado da arte da plataforma BIM (building information modeling), e se torna importante realizar essa análise com uma visão independente dos interesses comerciais que norteiam a literatura oferecida pelos fabricantes de software [1].

Diferentemente de um simples modelador 3D, a plataforma BIM é uma filosofia de trabalho que integra arquitetos, engenheiros e construtores (AEC) na elaboração de um modelo virtual preciso, o qual gera uma base de dados que contém tanto informações topológicas como os subsídios necessários para orçamento, cálculo energético e previsão das fases da construção, entre outras atividades.

Estudiosos afirmam ser o BIM é como um processo integrado que armazena e dinamiza a troca de informações de projetos e construção entre os stakeholders, criando modelos tridimensionais que representam as características físicas e funcionais do edifício e que tem um grande potencial no suporte de tomada de decisões em vários estágios do projeto [2].

As soluções BIM trabalham como gestores de banco de dados, de forma que qualquer alteração ou revisão realizada em qualquer parte de um modelo será automaticamente considerada em todas as demais formas de visualização da correspondente massa de dados e informações, sejam tabelas, relatórios ou desenhos (documentos), gerados a partir do modelo [3].

Embora o BIM exista há mais de vinte anos, apenas recentemente os proprietários de edifícios perceberam que o projeto, a construção e a operação podem ser feita de modo muito mais rápido e eficiente com sua utilização [4].

O uso desta tecnologia está se disseminado pelo setor da construção por ser um recurso para compartilhar informação sobre uma edificação e configurar uma base confiável para apoiar decisões e melhorar os processos no decorrer do ciclo de vida do projeto, baseando-se em uma representação digital de características físicas e funcionais dessa edificação [5].

Com o BIM os projetos são inevitavelmente mais detalhados, não somente devido às informações relacionadas ao modelo como também pela automatização dos seus elementos, contribuindo para a eliminação das causas referentes à escassez de detalhes de projetos, aos erros e às omissões. Estudiosos observam em suas pesquisas que o BIM propiciou um aumento da quantidade de informações disponíveis nos projetos realizados. Além disso, houve a geração de produtos antes não oferecidos ao cliente, como levantamento de quantitativos e o modelo virtual da edificação [6].

O BIM oferece uma tecnologia transformadora, por meio da sua capacidade de fornecer um recurso compartilhado digital para todos os participantes na gestão do ciclo de vida de um edifício desde o desenho preliminar, até o gerenciamento das facilidades e manutenções pós ocupacionais.

Como um banco de dados visual dos componentes do edifício, o BIM pode fornecer a quantidade exata e automatizada e ajudar na redução significativa da variabilidade das estimativas de custos [7].
As principais vantagens são a diminuição de erros de desenho e as facilidades nas alterações em projeto, devido à parametrização dos objetos, que permite a correção automática de cortes e vistas. Além disso, também é citado que a visualização em 3D melhora o entendimento dos participantes do projeto, trazendo soluções as dificuldades [8].

Outras vantagens são destacadas, como a maior velocidade na entrega do projeto, menor custo, maior produtividade por utilizar um único modelo, maior qualidade, por consequência nova oportunidades de receita e negócios, também maior foco no design e menor retrabalho [9].

\section{METODOLOGIA}

\section{II.1 - DELINEAMENTO}

Foi realizada uma revisão do conhecimento disponível na literatura científica, de artigos publicados entre 2000 e 2018, nas bases de dados Google Acadêmico. Foram selecionadas para a consulta nas bases de dados os seguintes descritores: BIM, Preparação, Vantagens.

\section{2 - SELEÇÃO DE TRABALHOS}

Os termos de busca utilizados nesta pesquisa foram agrupados como mostrados no Quadro 1:

Quadro 1: Termos utilizados na pesquisa.

\begin{tabular}{|c|c|c|c|}
\hline Escritor & Título & Ano & $\begin{array}{c}\text { O que aduz } \\
\text { sobre a } \\
\text { plataforma } \\
\text { BIM? }\end{array}$ \\
\hline BIM & $\begin{array}{l}\text { Breve histórico de } \\
\text { implantação da } \\
\text { plataforma BIM }\end{array}$ & 2011 & $\begin{array}{c}\text { O conceito de } \\
\text { BIM }\end{array}$ \\
\hline$-/ /-$ & $\begin{array}{c}\text { Sistema BIM: } \\
\text { análise da } \\
\text { percepção de duas } \\
\text { empresas }\end{array}$ & 2016 & $\begin{array}{c}\text { A praticidade } \\
\text { que a } \\
\text { plataforma } \\
\text { BIM oferece } \\
\text { à empresa } \\
\text { que a utiliza }\end{array}$ \\
\hline Preparação & $\begin{array}{l}\text { Metodologia BIM, } \\
\text { aplicada à } \\
\text { preparação, } \\
\text { controlo e gestão de } \\
\text { obra }\end{array}$ & 2012 & $\begin{array}{l}\text { A preparação } \\
\text { da obra com } \\
\text { a utilização } \\
\text { da plataforma } \\
\text { BIM }\end{array}$ \\
\hline Vantagens & $\begin{array}{l}\text { O uso do software } \\
\text { revit na Construção } \\
\text { Civil }\end{array}$ & 2016 & $\begin{array}{c}\text { A vantagem } \\
\text { que a } \\
\text { plataforma } \\
\text { BIM oferece }\end{array}$ \\
\hline$-/ /-$ & $\begin{array}{l}\text { Modelagem 5D } \\
\text { (BIM) - Processo de } \\
\text { orçamentação com } \\
\text { estudo sobre controle } \\
\text { de custos e valor } \\
\text { agregado para } \\
\text { empreendimentos de } \\
\text { Construção Civil }\end{array}$ & 2015 & $\begin{array}{c}\text { A redução de } \\
\text { custos que a } \\
\text { plataforma } \\
\text { BIM oferece }\end{array}$ \\
\hline
\end{tabular}

Fonte: Autores, (2019). 


\section{II.3 CRITÉRIOS PARA INCLUSÃO DE ARTIGOS:}

- Podem ser selecionados trabalhos acadêmicos realizados nos últimos 10 anos;

- Podem ser selecionados trabalhos acadêmicos que aduzam quanto à utilização da plataforma BIM na construção civil;

- Podem ser selecionados trabalhos acadêmicos que apresentem as vantagens oferecidas à quem faz uso da plataforma BIM;

- Podem ser selecionados trabalhos acadêmicos que aduzam como é feita a gestão da obra quando utilizada a plataforma BIM;

- Podem ser selecionados trabalhos acadêmicos que apresentem a conceituação histórica da plataforma BIM;

- Podem ser selecionados trabalhos acadêmicos que relatam as vantagens, como a redução de custos, da empresa que faz uso da plataforma BIM.

\section{II.4 CRITÉRIOS PARA EXCLUSÃO DOS ARTIGOS:}

- Não serão selecionados trabalhos acadêmicos realizados antes do ano 2000;

- Não serão selecionados trabalhos acadêmicos que não satisfaçam a nenhum critério de inclusão;

- Não serão selecionados trabalhos acadêmicos que aduzam critérios mínimos de importância da plataforma BIM;

- Não serão selecionados trabalhos acadêmicos que disponibilizam somente a metade do conteúdo do referido trabalho.

\section{II.5 ANÁLISE DE DADOS:}

\section{II.5.1 EMPRESA A:}

A empresa foi fundada em 1982 e localiza-se na cidade de São Leopoldo, atendendo a região do Vale do Rio dos Sinos.

O objetivo principal da empresa era a construção e a incorporação de imóveis para um público de classe média e baixa, onde a carência de ofertas era muito grande. Para tanto, a Empresa A identificou a necessidade de adequar seus produtos a esse tipo de mercado, atendendo às necessidades específicas desse tipo de cliente.

A empresa A possuía uma estrutura tendo em sua sede um escritório com a Diretoria e Comitê Gestor que está em constante comunicação com o Setor de Qualidade, onde se estabeleciam as normas e diretrizes efetivas, acompanhavam políticas das gerências e o planejamento organizacional, para que houvesse sucesso.

Organograma da empresa A:

- Diretoria de Projetos: Este setor utilizava BIM para alimentar de dados os outros setores da empresa, do planejamento à execução, contudo esta pesquisa apoiou-se diretamente nesta ramificação da empresa para obtenção das informações necessárias para o desenvolvimento do estudo.

- Diretoria de Novos Empreendimentos: setor responsável pelo apontamento de novos investimentos, e avaliação de terrenos, possuía o dirigente com maior experiência na empresa levando a uma grande visão de mercado.

- Execução: constituído por um gerente de execução que supervisionava 3 setores subordinados, um de execução das obras, onde se administrava a obra no canteiro, com um engenheiro, um auxiliar de engenharia, um estagiário, que coordenavam o técnico em edificações, o mestre de obras e o contramestre, e na obra possuía os pedreiros profissionais e seus serventes, e ainda os empreiteiros; o setor de planejamento e orçamento, responsável pelos materiais exigidos na obra e a necessidade de compra destes, no momento oportuno, em seu quadro havia um engenheiro e um auxiliar de engenharia; e o setor de pós-obra que proporcionava o acompanhamento após a entrega das edificações, encontrava-se neste setor um supervisor de pós-obra, um assistente administrativo, e um técnico em edificações.

- Diretoria Administrativa e Financeira: formada por um diretor com assistência jurídica e de contabilidade terceirizadas, e responsável por 5 setores.

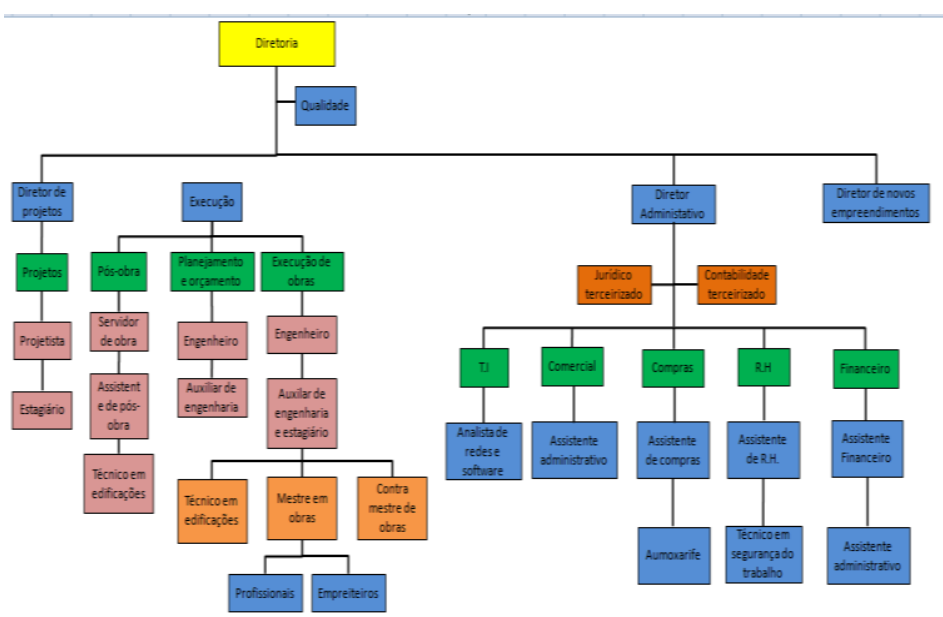

Figura 1: Organograma da empresa A. Fonte: Autores, (2019).

Sabedora de que a utilização da plataforma BIM traz muitos benefícios para a empresa que dela faz seu uso, foi que a empresa A optou por utilizar o Sistema BIM com a intenção de facilitar o desenvolvimento dos projetos e ter maior agilidade, e apresentar dados mais completos e confiáveis para os orçamentos, obtendo assim maior controle na execução de obras.

Após um estudo sobre os sistemas disponíveis no mercado, o escolhido para o perfil da empresa foi o Autodesk Revit, por se tratar de um dos mais conhecidos programas de modelagem do mercado da construção civil, por ser mais acessível e com a intenção de ter maiores ganhos na relação custo benefício, ocorrendo assim uma troca de plataforma, pois anteriormente era utilizado o Autodesk Autocad.

Durante o período de 2012 até novembro de 2015, utilizaram o software para realizar projetos padronizados da construtora, com a intenção de modernizar e qualificar estes projetos, e também porque alguns contratantes estavam exigindo que fosse usado um sistema em BIM, como é o caso da Petrobrás, e outras empresas seguiriam o seu exemplo, principalmente as ligadas ao Governo.

O fator de variação de preços, com a inflação, as mudanças financeiras no país e as incertezas dos fornecedores influenciaram 
no uso do sistema, na opinião do Diretor, que acabou sendo somente no projeto e não no gerenciamento da obra.

O Diretor deu ênfase a esta variação, que na prática colocariam um material no Revit que poderia não estar mais disponível na hora da execução, acabando com a lógica do BIM para o planejamento da obra.

$\mathrm{Na}$ época da coleta de dados, suas obras seguiam um padrão específico como mostra a Figura 2.

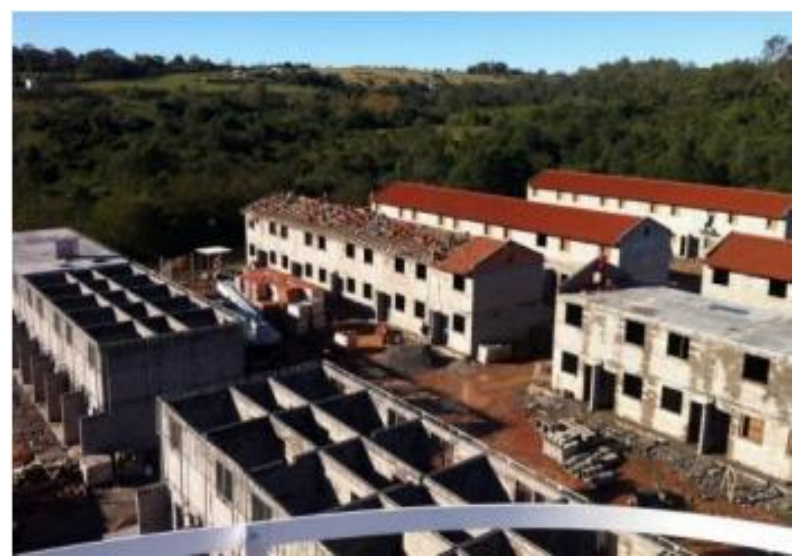

Figura 2: Empresa A. Fonte:

Mariana Cristina, (2014)

A previsão inicial da empresa A era que o sistema BIM levaria cinco anos para estar em pleno funcionamento, mas passou a dez anos após a implantação.

Entre as maiores vantagens que a empresa A esperava encontrar estão:

- A agilidade nas alterações do projeto, que eram necessárias no seu desenvolvimento e a visualização destes resultados;

- A precisão dos dados obtidos pelo sistema, como nos quantitativos de materiais de obra, e a confiabilidade destes dados;

- Após a execução dos projetos, gerando menores desperdícios proporcionando maior eficiência dos recursos da construtora.

Como maiores desvantagens observadas estão:

- A falta de banco de dados padronizados e completos, principalmente dos fornecedores de materiais para obra;

- A dificuldade de mão de obra qualificada para utilizar o sistema;

- O maior tempo de treinamento para adaptação dos profissionais que já trabalhavam nos projetos;

- A falta de interoperabilidade entre o sistema BIM e o que se usava para orçamentação na época o Revit, sendo necessária a alimentação separada dos quantitativos aumentando assim o tempo necessário de orçamento.

A mencionada empresa $\mathrm{A}$ acabou por não utilizar, até o momento da pesquisa, todos os recursos disponíveis nos sistemas BIM, no gerenciamento e práticas de planejamento, eles acreditavam não ser possível aplicar por falta de confiança na entrega dos materiais em obra, pois como poderia aplicar um conceito de Just in Time se os fornecedores não correspondiam as expectativas. Acabaram por utilizar somente os quantitativos para orçamentos.

\section{II.5.2 - EMPRESA B:}

À priori, temos que a estrutura de organização da empresa B é bastante diferenciada da da empresa A, vejamos na Figura 3.
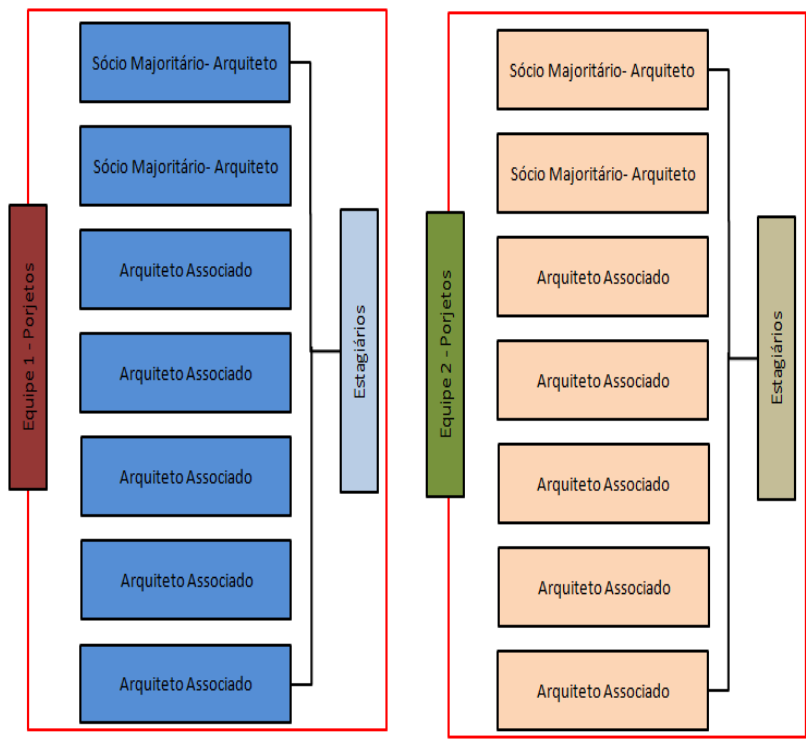

Figura 3: Organograma da empresa B. Fonte: Autores, (2019).

A empresa B trabalhava de forma colaborativa, com duas esquipes de projetos, sem uma estrutura rígida organizacional:

- Equipe 1: havia 2 sócios majoritários e 5 arquitetos associados, e os estagiários que variavam, onde um dos arquitetos ficava responsável em coordenar o restante.

- Equipe 2: havia 1 sócio majoritários e 6 arquitetos associados, e os estagiários que variavam, onde um dos arquitetos ficava responsável em coordenar o restante

A empresa B resolveu implantar um sistema baseado em tecnologia BIM em 2011, a partir deste momento, um dos proprietários se dedicou a aprender o software Revit e o ArchiCad com a intenção de conhecer e saber qual se adequaria melhor ao perfil da empresa.

No momento da pesquisa eram desenvolvidos somente os projetos arquitetônicos, os complementares eram feitos fora da empresa, porém eles recebiam estes projetos para fazer a compatibilização, alguns chegavam em AutoCad e eram importados para dentro do ArchiCad fazendo uma sobreposição de desenhos, assim verificavam as interferências. As empresas terceirizadas já estavam buscando utilizar sistemas BIM, que independentemente do software utilizado, trabalhando com a troca de arquivos IFC facilitaria a interoperabilidade dos dados.

A empresa B já estava totalmente adaptada ao uso do sistema BIM, acreditava ainda que era necessária atualização nos softwares para versões mais recentes, e treinamento para novas ferramentas.

Até o momento da pesquisa a única desvantagem observada era a falta de treinamento nos sistemas BIM dos novos profissionais que normalmente eram contratados, a necessidade de conhecimento já na Universidade. 
Para a empresa B houve diversas vantagens nesta mudança, como a facilidade em observar as interferências no projeto, e resolve-las com agilidade, a geração automática de vistas, cortes e fachadas, e alteração destas no momento em que se alterava a planta baixa, a qualidade muito superior na representação dos projetos, como na Figura 4 e a quantidade de detalhes de podiam ser inseridos no arquitetônico.

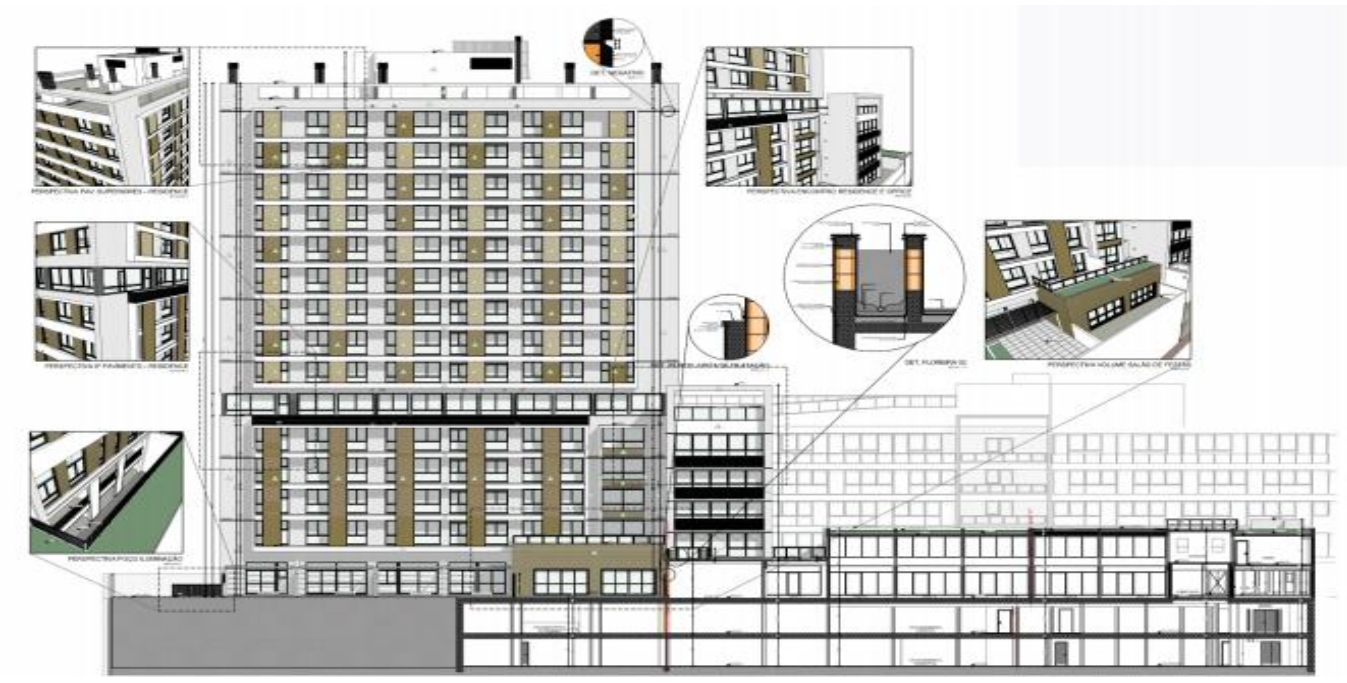

Figura 4: Empresa B.

Fonte: ArchiCad, (2018).

A figura 4 apresenta um corte desenvolvido na empresa B no software ArchiCad, com alguns itens detalhados, como floreiras e pingadeiras, e algumas perspectivas, como do poço de iluminação e do volume do salão de festas, que são geradas automaticamente.

$\mathrm{Na}$ empresa B, como demostrado pela figura 4, os sistemas BIM são utilizados para a modelagem dos projetos e geração de vistas, cortes, fachadas, toda documentação de forma mais detalhada e com maior qualidade e melhor visualização, porém não são aproveitas as diversas vantagens oferecidas pelos sistemas.

\section{PRINCIPAIS VANTAGENS NO USO DO SISTEMA BIM}

Tabela 1: Vantagens do uso do sistema BIM.

\begin{tabular}{|l|l|}
\hline \multicolumn{1}{|c|}{ Vantagens } & Referências Bibliográficas \\
\hline $\begin{array}{l}\text { Diminuição de erros de projeto, } \\
\text { geração de vistas, fachadas e } \\
\text { visualização 3D com agilidade e } \\
\text { realismo, possibilitando maior } \\
\text { produtividade, com foco no } \\
\text { design e menor retrabalho. }\end{array}$ & \\
\hline $\begin{array}{l}\text { Identificar e solucionar as } \\
\text { interferências e corrigi-las em } \\
\text { projeto, conduzindo a execução } \\
\text { edificação com maior } \\
\text { eficiência e segurança. }\end{array}$ \\
$\begin{array}{l}\text { ta } \\
\begin{array}{l}\text { Compatibilização dos projetos de } \\
\text { arquitetura com os demais, } \\
\text { identificando e corrigindo as } \\
\text { interferências ainda em projeto, } \\
\text { com automáticos e } \\
\text { visualização das estruturas. }\end{array}\end{array}$ \\
\hline
\end{tabular}

Fonte: Autores, (2019).

\section{PRINCIPAIS DESVANTAGENS NO USO DO SISTEMA BIM}

Tabela 2: Desvantagens do uso do sistema BIM.

\begin{tabular}{|l|l|}
\hline \multicolumn{1}{|c|}{ Desvantagens } & Referências Bibliográficas \\
\hline $\begin{array}{l}\text { Falta de compatibilidade com } \\
\text { outros softwares de outras } \\
\text { plataformas. }\end{array}$ & \\
\hline $\begin{array}{l}\text { O treinamento de mão de obra } \\
\text { para uso, por ainda ser pouco } \\
\text { difundido no mercado, existe } \\
\text { dificuldade em encontrar } \\
\text { pessoal qualificado. }\end{array}$ & $\begin{array}{l}\text { LYUZA, AMORIM E } \\
\text { LRESPO E RUSCHEL, } \\
\text { 200713. }\end{array}$ \\
\hline
\end{tabular}

Fonte: Autores, (2019).

\section{CONCLUSÃO}

Da revisão bibliográfica realizada para a elaboração do presente artigo, pode-se concluir que a utilização da plataforma BIM gera vantagens, essas de grande valia, para a empresa que a utiliza.

Vantagens observadas quando na utilização da plataforma BIM podem ser anotadas desde a redução de custos, até o tempo de conclusão da obra que está "no papel” para ser, assim, desenvolvida.

Comparando as informações obtidas com a revisão bibliográfica, somadas com as pesquisas feitas nas empresas A e $\mathrm{B}$, pode-se concluir que a falta de profissional habilitado para a 
utilização da plataforma BIM é a causa para o atraso do avanço do uso dessa tecnologia.

A utilização dos sistemas BIM em ambas empresas ainda era de baixa, pelo foco da empresa $B$, que não chegava ao canteiro de obras, e pela falta de profissionais treinados na empresa $\mathrm{A}$, que obteve maior dificuldade no uso.

De todo o exposto, temos que em um momento inicial a maior dificuldade é a mudança de paradigma, profissionais com mais tempo de trabalho, acostumados aos softwares, apresentam maior resistência e uma parte dos que estão entrando no mercado também não possuem o conhecimento necessário. O uso do BIM, se direcionado para as necessidades de controle de obra, pode trazer um planejamento com maior precisão, gerando uma melhoria no orçamento e facilidade da aplicação de técnicas como a do lean construction, porém não foi possível ver essas práticas no estudo.

\section{AGRADECIMENTOS}

A UNINORTE pelo apoio à Pesquisa.

\section{REFERÊNCIAS}

[1] Eastman, C. et al. BIM handbook: a guide to building information modeling for owners, managers, designers, engineers, and contractors. New Jersey - USA: John Wiley \& Sons, Inc., 2008.

[2] Meireles, A. R. Diga "Não" ao Hollywood BIM - Estratégia para uma integração avançada do BIM no processo construtivo. In: Seminário Internacional BIM - Modelagem da Informação da Construção, 4, 2013, São Paulo. Palestra. São Paulo: SINDUSCON, 2013. Disponível em: <http://www.sindusconsp.com.br/envios/2013/eventos/bim/Apres enta\%C3\%A7\%C3\%A3o_AntonioMeireles.pdf $>$. Acesso em 02/04/2019.

[3] Catelani, W. S. Fundamentos BIM. Coletânea implementação do BIM para construtoras e incorporadoras. Vol. 1. Câmara Brasileira da Indústria da Construção, Brasília, 2016. Disponível em:<http://cbic.org.br/bim/>. Acesso em 02/04/2019.

[4] Coates, P.; Arayici, Y.; Koskela, L.; Kagioglou, M.; Usher, C.;

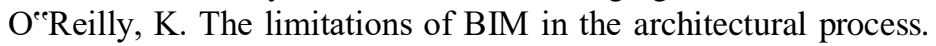
In: International Conference on Sustainable Urbanism, 1, 2010, Hong Kong. Anais... Hong Kong: International Conference on Sustainable Urbanization, 2010. Disponível em: <http://usir.salford.ac.uk/12898/2/PaulCoatesLimitationsofBIMIC SU.pdf>. Acesso em 03/04/2019.

[5] Nbims National Building Information Modeling Standard Version 1 - Part 1: Overview, Principles, and Methodologies. Washington, 2007. Disponível em: < http://www.wbdg.org/pdfs/NBIMSv1_p1.pdf $>$. Acesso em 03/04/2019.

[6] Vasconcelos, T. M. N. R. F. Building Information Model Avaliação do seu potencial como solução para os principais atrasos e desperdícios na construção portuguesa. 116f. Dissertação (Mestrado em Engenharia Civil), Faculdade de Ciências e Tecnologia da Universidade Nova Lisboa, 2010.
[7] Sabol, L. Challenges in cost estimating with Building Information Modeling. Design + Construction Strategies, 2008. Disponível em: <http://www.dcstrategies.net/files/2_sabol_cost_estimating.pdf>. Acesso em 03/04/2019.

[8] Souza, Livia L. Alves de; Amorim, Sérgio R. Leusin; Lyrio, Arnaldo de Magalhães. Impactos do Uso de BIM em Escritórios de Arquitetura: Oportunidades no Mercado Imobiliário, Gestão \& Tecnologia de Projetos. Periódico Científico do Instituto de Arquitetura e Urbanismo da USP. São Paulo. 2009.

[9] Justi, Alexander Rodrigues. Implantação da Plataforma Revit nos Escritórios Brasileiros: Relatos de Uma Experiência. Gestão \& Tecnologia de Projetos. UNIBENNET, Rio de Janeiro, 2008.

[10] Martins, João Poças; MAIA Andreia, Bruno; Santos Diogo; Neves João; Martins, Margarida. BIM: O que é? Portugal. Mestrado Integrado em Engenharia Civil, Universidade do Porto. 2013.

[11] Bortolotto; Mariana Cristina. Compatibilização de projetos de uma habitação: Verificação de incompatibilidades no sistema de projetação 2D e na modelagem 3D. Universidade Federal de Santa Catarina. Florianópolis. 2014.

[12] Chagas; Leila Soares Viegas Barreto. Padilha; Marcos Antônio Junior. Teixeira; Eduardo da Cruz. Gestão da Tecnologia: Uso do sistema BIM para a compatibilização de projetos. XXXV Encontro Nacional de Engenharia de Produção. Perspectivas Globais para a Engenharia de Produção. Fortaleza. CE. Outubro de 2015.

[13] Crespo, Cláudia Campos; Ruschel, Regina Coeli. Ferramentas BIM: um desafio para a melhoria no ciclo de vida do projeto. III Encontro de Tecnologia de Informação e Comunicação na Construção Civil. Porto Alegre. 2007.

[14] Koelln, Friedrich Pfeifer. Tecnologia BIM na Construção Civil: Composição de Custo Direto. Universidade Federal do Rio Grande do Sul. Porto Alegre. 2015.

[15] Menezes, Gilda Lúcia Bakker Batista de. Breve histórico de implantação da plataforma BIM. A brief historical of the BIM platform implementation. Cadernos de Arquitetura e Urbanismo. 2011. Disponível em:< http://periodicos.pucminas.br/index.php/Arquiteturaeurbanismo/a rticle/viewFile/3363/3719>. Acesso em: 05/04/2019.

[16] Riske, Joseandra. Sistemas BIM: análise da percepção de duas empresas. Santa Rosa. 2016. Disponível em: <http://bibliodigital.unijui.edu.br:8080/xmlui/bitstream/handle/12 3456789/3401/TCC\%20JOSEANDRA\%20RISKE.pdf?sequence $=1>$. Acesso em: 06/04/2019.

[17] Esteves, Armando. Metodologia BIM, aplicada à preparação, controlo e gestão de obra. 2012. Disponível em: <file:///D:/Downloads/DM_ArmandoEstevesSilva_2012_MEC.pd f> . Acesso em: 06/04/2019. 
[18] Souza, Flávia Ribeiro. MARTINS, Samuel Lima. O uso do software revit na construção civil. Anais VIII. SIMPAC - Volume 8 - n. 1. Viçosa/MG. 2016. Disponível em:< file:///D:/Downloads/714-1013-1-PB.pdf>. Acesso em: 07/04/2019.

[19] Sakamori, Marcelo Mino. Modelagem 5d (BIM) - processo de orçamentação com estudo sobre controle de custos e valor agregado para empreendimentos de construção civil. Curitiba. 2015. Disponível

em:

<https://www.acervodigital.ufpr.br/bitstream/handle/1884/41394/ R\%20-\%20D\%20-

\%20MARCELO\%20MINO\%20SAKAMORI.pdf?sequence=2\&i sAllowed $=y$ > . Acesso em: 08/04/2019. 